\title{
Optimal induction treatment regimens for extranodal NK/T-cell lymphoma: lessons learned, challenges, and proposals
}

\author{
Jing Yang ${ }^{\mathrm{a}}$, Ruiying Fu ${ }^{\mathrm{a}}$, Xindi Liu ${ }^{\mathrm{a}}$, Liqiang Wei ${ }^{\mathrm{a}}$, Liang Wang ${ }^{\mathrm{a}, \mathrm{b}, *}$ \\ ${ }^{a}$ Department of Hematology, Beijing Tongren Hospital, Capital Medical University, Beijing 100730, China. \\ ${ }^{b}$ Beijing Advanced Innovation Center for Big Data-Based Precision Medicine, Beihang University \& Capital Medical \\ University, Beijing Tongren Hospital, Beijing 100730, China.
}

Extranodal NK/T-cell lymphoma (ENKTCL) is a highly aggressive and Epstein-Bar virus (EBV)-associated hematologic malignancy, most prevalent in the Far East and South America [1]. No standard treatment strategy has been defined. It is well recognized that combination of asparaginase-based chemotherapy (CT) and radiotherapy (RT) benefit early-stage ENKTCL. It has been validated that upfront RT can cure most stage I patients of ENKTCL without risk factors, and asparaginase (either L-asparaginase or pegaspargase) is the backbone of chemotherapy for ENKTCL. In recent years, a variety of asparaginasebased regimens have been found to be effective in earlystage ENKTCL, such as GELOX (gemcitabine, oxaliplatin, and asparaginase) [2] or EPOCHL (etoposide, prednisone, vincristine, cyclophosphamide, doxorubicin, and asparaginase) [3], but with different toxicities. Thus, we are wondering how much is too much for early-stage ENKTCL patients, and is intensive chemotherapy really necessary? Moreover, the international T-cell Project reported the updated survival outcomes of a large cohort of worldwide patients with ENKTCL (except Chinese) this year, and more than 60 regimens were used in this report, indicating a lack of standard treatment regimens [4]. Compared with the survival data of Chinese patients with ENKTCL, the outcomes reported by this international multi-center cohort seem unsatisfactory. Due to very small number of patients enrolled from the USA and no patients from China, it is of importance to show the data from these two large countries, which enable us to depict the real-world data of ENKTCL globally. In this multi-center retrospective study, we compared the efficacy, long-term survival outcomes, and safety profiles between GELOX

\footnotetext{
* Corresponding author: Liang Wang

Mailing address: Department of Hematology, Beijing Tongren Hospital, Capital Medical University, Beijing 100730, China. Email: wangliangtrhos@126.com
}

Received: 23 November 2020 / Accepted: 16 December 2020 and EPOCHL regimen in early-stage ENKTCL patients. Meanwhile, the survival outcomes of all-stage patients from China and SEER (Surveillance, Epidemiology, and End Results) database were compared.

A total of 494 patients of ENKTCL with all stages (AnnArbor stage I, $n=224$; stage II, $n=168$; stage III-IV, $n=102$ ) from Beijing Tongren Hospital were included in this study, and all patients received asparaginasebased treatment, among whom 193 patients with stage I-II ENKTCL were treated with 4-6 cycles of GELOX (pegaspargase $2,500 \mathrm{IU} / \mathrm{m}^{2} \mathrm{~d} 1$, gemcitabine $1,000 \mathrm{mg} / \mathrm{m}^{2}$ $\mathrm{d} 1,8$, oxaliplatin $100 \mathrm{mg} / \mathrm{m}^{2} \mathrm{~d} 1$, repeated every 3 weeks) or EPOCHL (etoposide $50 \mathrm{mg} / \mathrm{m}^{2} / \mathrm{d}$, vincristine $0.5 \mathrm{mg} / \mathrm{d}$, doxorubicin $10 \mathrm{mg} / \mathrm{m}^{2} / \mathrm{d}$ were administered by continuous intravenous infusion over $96 \mathrm{~h} \mathrm{d1-4}$, prednisone $60 \mathrm{mg} /$ $\mathrm{m}^{2} / \mathrm{d}$ was administered orally on days $1-5$, cyclophosphamide $750 \mathrm{mg} / \mathrm{m}^{2}$ was given as an intravenous bolus on day 5 , L-asparaginase $10,000 \mathrm{U} / \mathrm{d}$ was administered by intravenous infusion on days 6-10 or pegaspargase 2500 $\mathrm{IU} / \mathrm{m}^{2} \mathrm{~d} 1$, repeated every 3 weeks) regimen combined with radiotherapy ( $\geq 50 \mathrm{~Gy}$ ), either sequential or sandwich chemoradiation. Response to treatment was evaluated using MRI scan or PET-CT scan after chemotherapy and end of treatment. Progression free survival (PFS) was calculated from diagnosis of ENKTCL to disease progression, death of any reason, or last follow-up, whichever came first. Overall survival (OS) was defined from diagnosis to death of any reason, or last follow-up, whichever came first. The institutional ethics review board approved this study and all patients provided informed consent for publication of clinical data at their first visit, but all patients in this study had been de-identified. Moreover, we identified 1140 patients with ENKTCL from the SEER database (www.seer.cancer.gov) based on the ICD-O-3 code for histology (9719, ENKTCL) from 1987 to 2015, and 330 patients were excluded from our analysis due to unknown stage or missing detailed survival data.

68 patients with early stage ENKTCL received GELOX induction therapy and 125 patients were treated with EPOCHL regimen. No significant difference was found between those two groups concerning baseline character- 
istics. At the end of treatment, a complete response (CR) rate of $75 \%$ and an overall response rate (ORR) of $98.5 \%$ was achieved in the GELOX group, and the CR rate and ORR were $56 \%$ and $94.4 \%$ in the EPOCHL group, respectively. Although there was no significant difference in ORR between GELOX and EPOCHL regimen, as is shown in Figure 1, patients treated with GELOX had significantly better PFS and OS than those with EPOCHL $(P<0.05)$. Meanwhile, the safety profile of GELOX regimen was more favorable than EPOCHL, especially the hematology toxicities. As expected, patients with stage II disease had significantly inferior outcomes than those with stage I disease $(P<0.05)$. The end of treatment efficacy had a significant impact on the long-term survival outcomes, as patients with CR enjoyed more favorable PFS and OS than those without CR $(P<0.05)$. In the GELOX cohort, efficacy assessment data was available before RT consolidation. As is shown in Figure 1-H, patients without $\mathrm{CR}$ after induction chemotherapy had significantly inferior PFS than those with $\mathrm{CR}(P=0.033)$, though part of those patients could be salvaged by RT consolidation and attained CR at the end of RT.

The detailed baseline characteristics of both Chinese and SEER cohorts were shown in Table 1. More patients in the SEER database were treated before 2010 when asparaginase-based therapies were not well recognized. More-

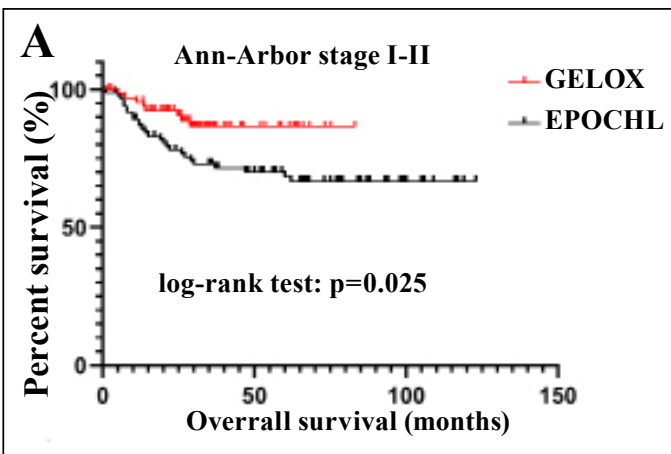

C

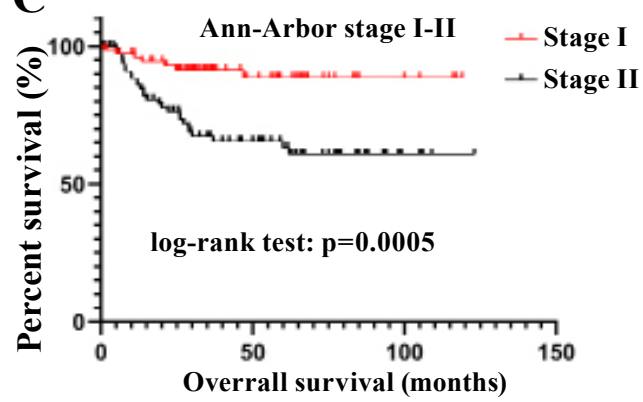

$\mathbf{E}$

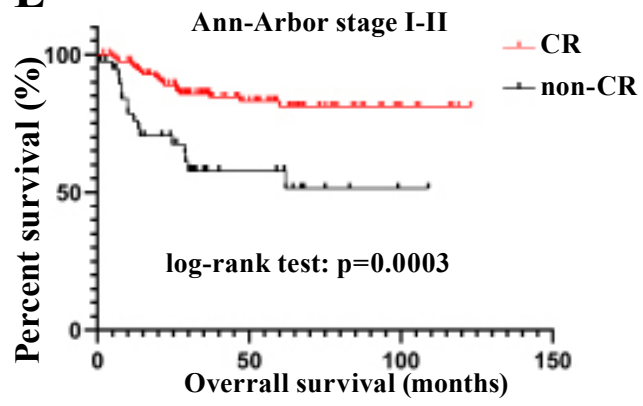

G

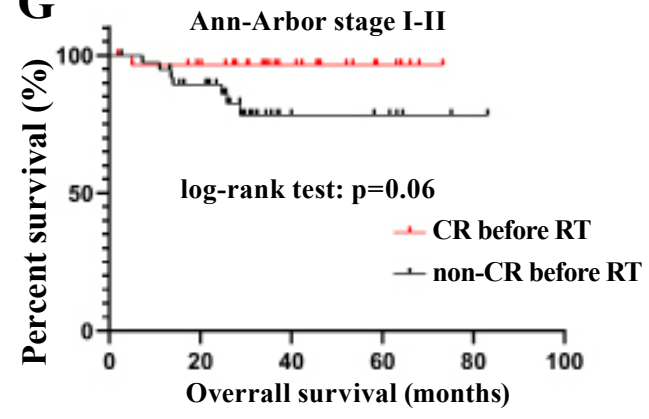

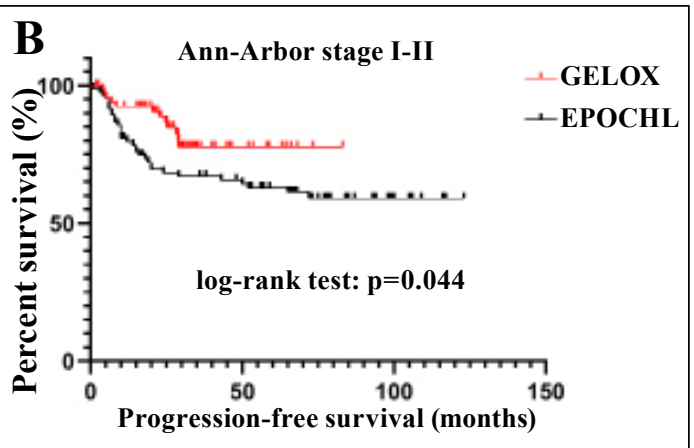

D

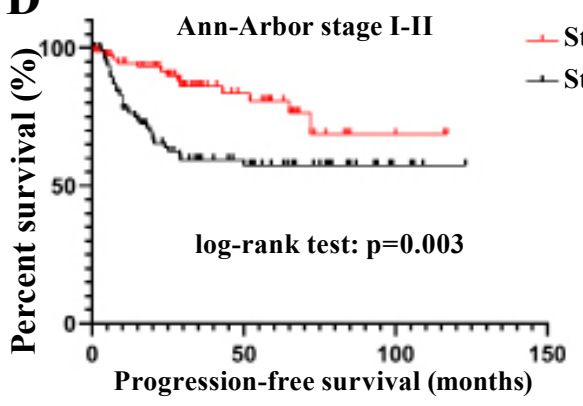

F
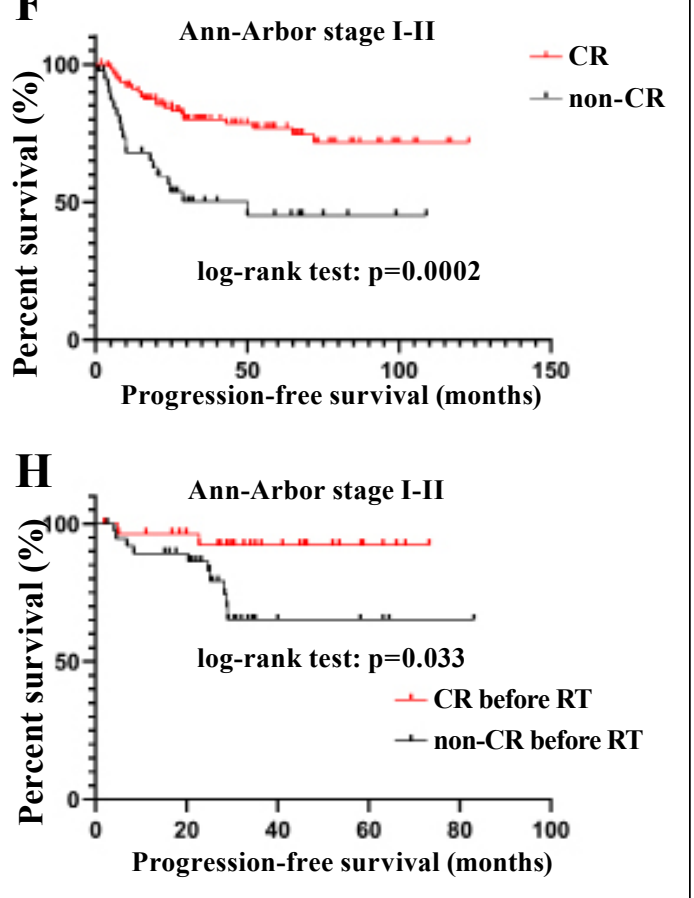

Figure 1. Survival curves for ENKTCL patients with localized disease. Patients treated with GELOX regimen had significantly better OS (A) and PFS (B) than those treated with EPOCHL. Patients with stage II disease had significantly inferior OS (C) and PFS (D) than those with stage I. The end of treatment response significantly correlated with prognosis of ENKTCL (E, F). For patients treated with GELOX, CR before radiotherapy indicated significantly better PFS (H) and a trend for better OS (G). CR means complete response, RT means radiotherapy. 


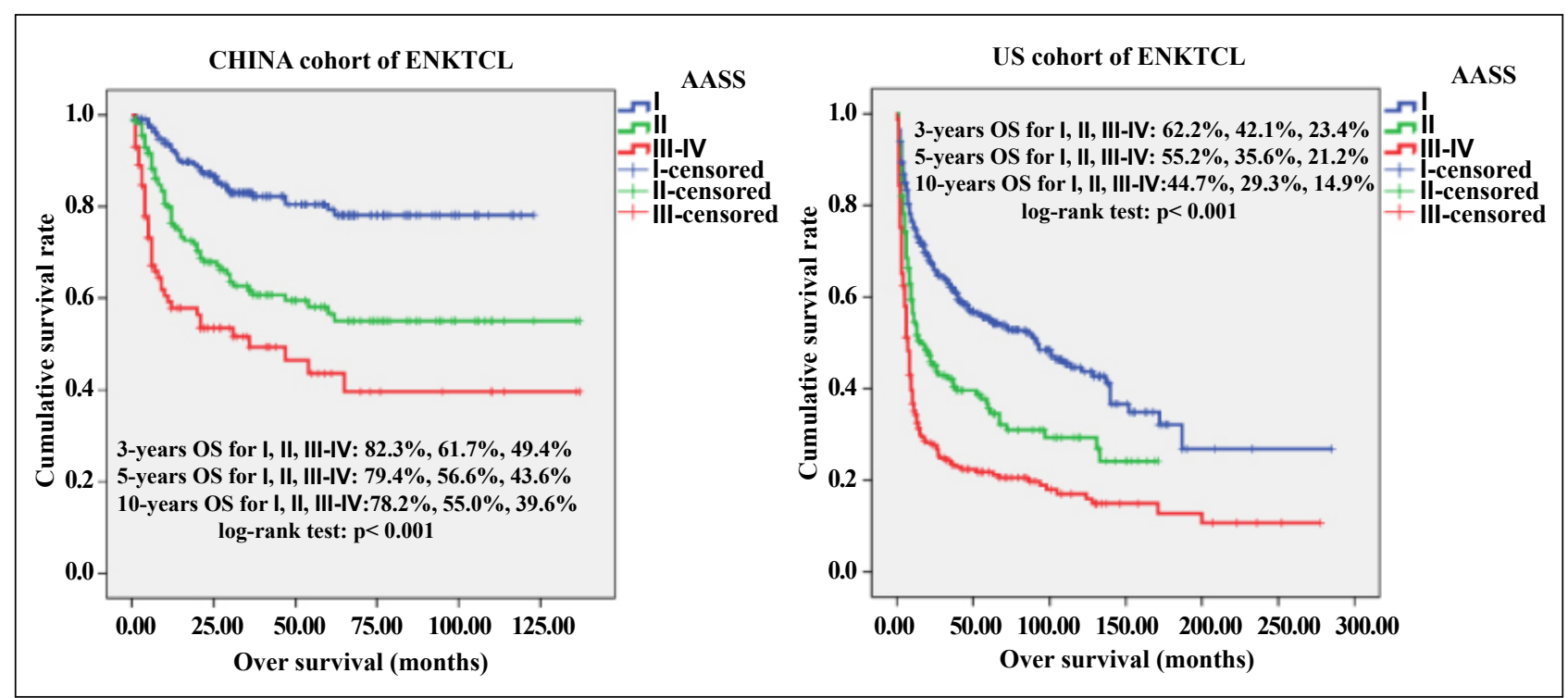

Figure 2. Survival curves for patients of ENKTCL in the Chinese (A) and SEER (B) cohorts. AASS means Ann-Arbor staging system.

Table 1. The baseline characteristics of ENKTCL patients.

\begin{tabular}{lccc}
\hline & $\begin{array}{c}\text { Chinese cohort } \\
(N=\mathbf{4 9 4})\end{array}$ & $\begin{array}{c}\text { SEER database } \\
(N=\mathbf{2 4 1})\end{array}$ & $P$-value \\
\hline Year of diagnosis & & & \\
$1987-2009$ & $191(38.7 \%)$ & $443(54.7 \%)$ & $P<0.001$ \\
2010 -now & $303(61.3 \%)$ & $367(45.3 \%)$ & \\
Gender & & & \\
Male & $335(67.8 \%)$ & $524(64.7 \%)$ & $P=0.274$ \\
Female & $159(32.2 \%)$ & $286(35.3 \%)$ & \\
Age & & & \\
$<60$ & $403(81.6 \%)$ & $513(63.3 \%)$ & $P<0.001$ \\
$\geq 60$ & $91(18.4 \%)$ & $297(36.7 \%)$ & \\
Ann-Arbor stage & & & \\
I & $179(36.2 \%)$ & $381(47.0 \%)$ & \\
II & $197(39.9 \%)$ & $179(22.1 \%)$ & $P<0.001$ \\
III-IV & $118(23.9 \%)$ & $250(30.9 \%)$ & \\
Primary lymphoma site & & & \\
UADT & $452(91.5 \%)$ & $596(73.6 \%)$ & $P<0.001$ \\
Non-UADT & $42(8.5 \%)$ & $214(26.4 \%)$ & \\
Nasal cavity & $320(64.8 \%)$ & $387(47.8 \%)$ & $P<0.001$ \\
Extra-nasal sites & $174(35.2 \%)$ & $423(52.2 \%)$ & \\
\hline & & & \\
\hline
\end{tabular}

over, significantly more patients in the SEER database had extra-nasal or non-upper aerodigestive tract (UADT) disease. The treatment strategies were unknown for the SEER database, and asparaginase-based chemotherapy with or without combined radiotherapy was administrated in the Chinese cohort. As is shown in Figure 2, the five-year OS rate was $79.4 \%, 56.6 \%$, and $43.6 \%$ for patients with stage I, II, and III-IV, respectively, in the Chinese cohort, which seemed much better than that in the SEER database $(55.2 \%$, $35.6 \%$, and $21.2 \%$, respectively). This discrepancy may be caused by unbalanced baseline characteristics and different treatment strategies. Also, the etiology and biology of ENKTCL may differ between Asian and Western countries.

Due to the distinct geographic pattern of ENKTCL, many studies were done in Asian countries, and asparaginasebased induction therapies were well recognized, should be given in the first-line setting. Nowadays, risk-model guided therapy should be implemented in clinical practice [5]. For those localized diseases at low-risk, radiotherapy alone could achieve satisfactory results, and asparaginasebased chemotherapy should be added to radiotherapy for those with risk factors. The standard induction regimens were not defined yet, and our previous study demonstrated that pegaspargase monotherapy in combination with concurrent radiotherapy could attain $100 \%$ CR rate for early stage disease [6], which needs to be validated in phase 3 RCTs. Although the prognosis is much better for the Chinese cohort, patients with the advanced disease still fare poorly. Increasing evidence has shown the advantage of immunotherapy (PD-1/PD-L1 blockade) in relapsed/ refractory ENKTCL [7], and we guess the first-line use of immunotherapy in combination with asparaginasebased chemotherapy might further improve the prognosis of patients with advanced disease [8]. Recently, a novel molecular subtyping system was defined for ENKTCL, including TSIM, MB, and HEA subtypes, with both prognostic value and treatment guidance [9]. In the future, prospective clinical trials should be conducted incorporating the new risk models and molecular subtyping system, to further implement precision medicine in ENKTCL.

\section{Declarations}

Financial support and sponsorship: None.

Conflicts of interest: Liang Wang is a member of the Editorial Board of Aging Pathobiology and Therapeutics. All authors declare no conflict of interest and were not involved in the journal's review or desicions related to this manuscript.

Ethical approval and consent to participate: Not applicable.

\section{References}


1. Wang L, Li L R, Zhang L, et al. The landscape of new drugs in extranodal NK/T-cell lymphoma. Cancer Treatment Reviews, 2020, 89

2. Wang L, Wang Z, Chen X, et al. First-line combination of gemcitabine, oxaliplatin, and L-asparaginase (GELOX) followed by involved-field radiation therapy for patients with stage IE/IIE extranodal natural killer/T-cell lymphoma. Cancer, 2013, 119(2): 348-355.

3. Wei L, Wang J, Ye J, et al. Long-term outcomes of patients treated with an EPOCHL regimen as first-line chemotherapy for newly diagnosed extranodal natural killer/ T-cell lymphoma: a retrospective single-center study. Leukemia \& Lymphoma, 2020, 61(2): 337-343.

4. Fox C P, Civallero M, Ko Y H, et al. Survival outcomes of patients with extranodal natural-killer T-cell lymphoma: a prospective cohort study from the international T-cell Project. The Lancet Haematology, 2020.
5. Chen S Y, Yang Y, Qi S N, et al. Validation of nomogramrevised risk index and comparison with other models for extranodal nasal-type NK/T-cell lymphoma in the modern chemotherapy era: indication for prognostication and clinical decision-making. Leukemia, 2020: 1-13.

6. Wang H, Wang L, Li C, et al. Pegaspargase Combined with Concurrent Radiotherapy for Early-Stage Extranodal Natural Killer/T-Cell Lymphoma, Nasal Type: A Two-Center Phase II Study. The Oncologist, 2020, 25(11): e1725.

7. Wang L, Wang J. PD-1 blockade in extranodal NK/T-cell lymphoma: who is in charge?. Leukemia, 2020: 1-2.

8. Wang L, Wang J. Extranodal natural-killer T-cell lymphoma: experience from China. The Lancet Haematology, 2020, 7(6): e441.

9. Xiong J, Cui B W, Wang N, et al. Genomic and Transcriptomic Characterization of Natural Killer T Cell Lymphoma. Cancer Cell, 2020, 37(3): 403-419. e6.

Cite this article as: Yang J, Fu R, Liu X, et al. Optimal induction treatment regimens for extranodal NK/T-cell lymphoma: lessons learned, challenges, and proposals[J]. Aging Pathobiology and Therapeutics, 2020, 2(4): 226229. 